\title{
Correction to: Contraception, Menstruation, and Sexuality after Bariatric Surgery: a Prospective Cohort Study
}

\author{
Julie Luyssen ${ }^{1} \cdot$ Goele Jans ${ }^{2,3}$ - Annick Bogaerts ${ }^{2,3,4}$ - Dries Ceulemans ${ }^{5} \cdot$ Christophe Matthys $^{6,7}$. \\ Bart Van der Schueren ${ }^{6,7}$. Matthias Lannoo ${ }^{6,8}$. Johan Verhaeghe ${ }^{2,5}$. Luc Lemmens ${ }^{9}$. Lore Lannoo ${ }^{5}$. \\ Jill Shawe ${ }^{10} \cdot$ Roland Devlieger ${ }^{2,5,11}$
}

Published online: 14 December 2017

(C) Springer Science+Business Media, LLC, part of Springer Nature 2017

\author{
Correcttion to: Obes Surg \\ https://doi.org/10.1007/s11695-017-3033-7
}

The spelling of author name Jill Shawe was incorrect in the original article. It is correct here. The original article was corrected.

The online version of the original article can be found at https://doi.org/ 10.1007/s11695-017-3033-7

\section{Roland Devlieger}

roland.devlieger@uzleuven.be

1 Master of Family Medicine, KU Leuven, Leuven, Belgium

2 Department of Development and Regeneration, KU Leuven, Herestraat 49, 3000 Leuven, Belgium

3 Faculty of Health and Social Work, Research Unit Healthy Living, UC Leuven-Limburg, Leuven, Belgium

4 Faculty of Medicine and Health Sciences, Centre for Research and Innovation in Care (CRIC), University of Antwerp,

Antwerp, Belgium

5 Department of Obstetrics and Gynecology, University Hospitals Leuven, Herestraat 49, 3000 Leuven, Belgium
6 Department of Clinical and Experimental Medicine, KU Leuven, Herestraat 49, 3000 Leuven, Belgium

7 Department of Endocrinology, University Hospitals Leuven, Herestraat 49, 3000 Leuven, Belgium

8 Department of Abdominal Surgery, University Hospitals Leuven, Herestraat 49, 3000 Leuven, Belgium

9 Department of Abdominal Surgery, St-Niklaas Hospital, Moerlandstraat 1, 9100 St-Niklaas, Belgium

10 Faculty of Health and Medical Sciences, University of Surrey, Guildford, Surrey GU2 7XH, UK

11 Department of Obstetrics, Gynecology and Reproduction, St-Augustinus Hospital Wilrijk, Oosterveldlaan 24, 2610 Wilrijk, Belgium 